\title{
Opioidtherapie
}

\section{Aus der Schmerzspirale ausbrechen}

"N Noch immer scheuen sich einige Ärzte und Patienten vor dem Gebrauch von Opioiden in der Schmerztherapie. Um jedoch einer Chronifizierung entgegenzuwirken, ist eine individualisierte, medikamentöse Behandlung mit Opioiden oft der erste Schritt.

Bis zu 15 Millionen Menschen in Deutschland leiden unter chronischen Schmerzen. Unbehandelt lassen diese jede Bewegung schmerzen, was oft zu einer Vermeidungshaltung und damit zu Bewegungsmangel führt. Und schon befindet sich der Patient in einer Schmerzspirale, aus der er mit Hilfe einer multimodalen Therapie ausbrechen müsse, erklärte Dr. med. Sabine Hesselbarth, niedergelassene Fachärztin für Anästhesie und spezielle Schmerztherapie in Mainz, auf einem Symposium der Firma Mundipharma in Frankfurt. „Häufig ist die medikamentöse Therapie mit Opioiden der erste Schritt, um eine multimodale Therapie überhaupt beginnen zu können“, sagte Hesselbarth und verdeutlichte die Komplexität der Schmerztherapie am Beispiel der Patientin Frau C. Diese kam 2009 im Alter von 53 Jahren mit dumpfen, ziehenden, brennenden Dauerschmerzen im unteren Rücken zu der Schmerzmedizinerin. Außerdem litt sie unter Komorbiditäten wie
Gonarthrose beidseitig mit Knieprothese rechts, Adipositas, Nikotinabusus und eine koronare Herzerkrankung. Auch psychovegetative Beschwerden wie Schwindel, Schlaf- und Konzentrationsstörungen beschrieb die Patientin. Die bisherige Therapie umfasste bis zu fünfmal täglich 600 mg Ibuprofen sowie Novaminsulfon bei Bedarf.

\section{Opioiddosis optimieren}

„Ziel einer Schmerztherapie ist auch die Korrektur einer Fehltherapie“, verdeutlichte Hesselbarth und erklärte, sie habe bei Frau C. zunächst die NSAR abgesetzt und eine Opioidtherapie mit zweimal täglich 20 mg Oxycodon unter Antiemetikum begonnen und nachts zusätzlich Mirtazapin 7,5-15 mg und Novaminsulfon angesetzt. Dies führte zu einer Reduktion der Schmerzstärke auf der numerischen Rating Skala (NRS; 0= kein Schmerz, $10=$ stärkste vorstellbare Schmerzen) von 8-9/10 auf 6/10 NRS. Auch Psychotherapie, Physiotherapie und RehaSport waren im multimodalen Setting enthalten.

Ein wichtiger Bestandteil der Therapie sei außerdem die regelmäßige Kontrolle der Effektivität der Maßnahmen, betonte Hesselbarth. So habe ihre Pati- entin trotz Laxanzien über anhaltende Obstipation geklagt, so dass die Anästhesistin die Behandlung auf zweimal täglich 20 mg Oxycodon/10mg Naloxon $\left(\operatorname{Targin}^{\circledR}{ }^{\circledR}\right.$ umstellte. Die Kombination mit Naloxon erhält die normale Darmfunktion und die Patienten leiden weniger an Übelkeit, Erbrechen und Schwindel.

Die Fixkombination aus retardiertem Oxycodon und retardiertem Naloxon ist zur Behandlung von starken Schmerzen, die nur mit Opioid-Analgetika ausreichend behandelt werden können, wie Bewegungs-, neuropathischen und Tumorschmerzen zugelassen. Die vier erhältlichen Wirkstärken ermöglichen eine individuelle Dosisanpassung, wobei die Standard-Anfangsdosierung bei $10 \mathrm{mg} / 5 \mathrm{mg}$ liegt und eine Tagesdosis von $2 \times 80 \mathrm{mg} / 40$ mg nicht überschritten werden sollte. Häufige Nebenwirkungen sind Kopfschmerz, Hitzewallungen, Abdominalschmerzen und Hautausschlag.

(ajr)

Pressegespräch „Opioide - Dosisoptimierung bei chronischen Schmerzen"; im Rahmen des Deutschen Schmerzund Palliativtages 2017. Frankfurt/M., 24.3.2017 (Veranstalter: Mundipharma GmbH Deutschland)

\section{Blutzuckerkontrolle digital}

\section{Move...App erhält den Innovationspreis-IT 2017}

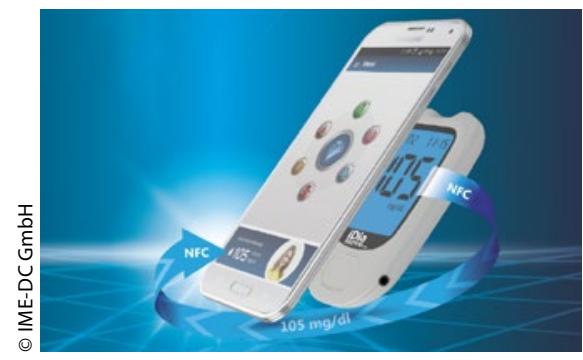

") Mit der Verwendung der immer mehr genutzten NFC (Near Field Communication) Technologie wird eine einfache und sichere Übertragung auch der Blutzuckermesswerte von ei- nem Blutzuckermessgerät auf ein Smartphone oder Tablet ermöglicht. Die Technologie erlaubt es durch bloßes Aneinanderhalten der Geräte jederzeit, überall und schnell die Daten zu übermitteln. Ein störendes Verbindungskabel oder zeitaufwendiges Pairing - wie bei Bluetooth üblich - sind nicht mehr notwendig.

Alle Diabetiker weltweit, egal ob jung oder alt, selbst sehbehinderte Menschen können mit Hilfe der move...App ihre gemessenen Blutzuckerwerte einfacher dokumentieren und mitteilen. Mit move...und der dazugehörigen Webplattform wird die
Lebensqualität des Diabetikers als auch dessen Behandlungsmöglichkeit durch einen Facharzt entscheidend verbessert. Über das Webportal findet der Facharzt schnell und übersichtlich sämtliche für die Behandlung relevanten Patientendaten vor. Diese kann der Facharzt für eine stetige Optimierung des Patiententherapieplans nutzen. Zusätzlich ermöglicht das System den Krankenkassen ein effizienteres, ressourcensparenderes Gesundheitsmanagement.

www.idiamove.de / www.imedc.de 